»verstorbene[n] Dr. Goebbels« zu vergleichen, der sich »derselben Mittel« bei seiner "Hetzerei gegen die Sowjetunion « bediente. ${ }^{236}$

Der zwischen der sowjetischen Delegation und Lasky im Oktober 1947 auf dem ersten gesamtdeutschen Schriftstellerkongress in Berlin ausgetragene Streit war ein Symptom für den ausbrechenden Kalten Krieg, der dann mit der Berlinkrise im darauf folgenden Jahr seinen ersten Höhepunkt fand. Der Kongress besiegelte zudem symbolisch die Teilung der deutschen Intellektuellen entlang der Frontlinien des Ost-West-Konfliktes. Der im Oktober in Berlin verabredete zweite gesamtdeutsche Schriftstellerkongress, der im Mai 1948 in Frankfurt a. M. stattfand, war bereits ausdrücklich durch den existenziellen Gegensatz zwischen den beiden Supermächten geprägt und ging ohne die ostdeutschen Autoren über die Bühne. ${ }^{237}$

Die Versuche der Sowjetunion durch den Kulturbund im Rahmen einer grundsätzlichen antifaschistisch-»demokratischen« Kulturpolitik in Deutschland, nationalneutralistische Kreise und friedensorientierte Linksintellektuelle für sich zu gewinnen, um die beiden miteinander verbundenen Ziele zu erreichen - die Spaltung der deutschen Nation zu verhindern und das eigene politische System in Deutschland einzuführen -, führte nicht nur zur Aufspaltung der deutschen Schriftsteller. Vor dem Hintergrund der »Zwei-Lager-Theorie« Schdanows und dem In-Gang-Kommen der antiamerikanischen Propaganda der Kominform - wie er zum Beispiel auf dem denkwürdigen ersten deutschen Schriftstellerkongress durch die sowjetische Delegation zum Ausdruck kam - waren die ideologischen und mithin die politischen Fronten geklärt. Aufseiten der amerikanischen Besatzungsbehörde setzten seit Oktober 1947 mit Blick auf die künftige Deutschlandpolitik vermehrt konkrete Planungen ein, die dieser Situation Rechnung trugen. Die Gründung der Zeitschrift Der Monat im Oktober 1948 war ein unmittelbares Resultat ebendieser Planungen.

\title{
4.3 Die Gründung und die Funktion des Monat
}

Im Rahmen seiner auf einer umfangreichen Quellenbasis beruhenden Studie Freiheit in der Offensive? Der Kongreß für kulturelle Freiheit und die Deutschen geht Hochgeschwender auch auf die facettenreichen Aspekte der Gründung und der Funktion der Zeitschrift Der Monat ein. ${ }^{238}$

Danach kündigte der amerikanische Militärgouverneur Clay angesichts der sich zuspitzenden politischen Situation in Deutschland auf einer Pressekonferenz am 28. Oktober $1947^{239}$ in Berlin die Operation »Talk Back« an, mit der unter genuin antikom-

236 Siehe die Replik von Valentin Katajew, in: Ebd., S. 336 f. (Zitate S. 336).

237 So Waltraud Wende-Hohenberger (Hg.), Der erste gesamtdeutsche Schriftstellerkongreß nach dem Zweiten Weltkrieg im Ostsektor Berlins vom 4. bis 8. Oktober 1947, Frankfurt a. M. u. a. 1988, S. XXVIII u. XXIX (Vorwort).

238 Siehe Hochgeschwender, Freiheit in der Offensive?, S. 139-203. Die nachfolgenden Ausführungen beziehen sich in erster Linie auf die Untersuchungsergebnisse dieser Studie, die neben Archivmaterial auch die zurzeit verfügbare Forschungsliteratur einbezieht. Es wird im Folgenden darauf verzichtet, die von ihm verwendeten Quellen jeweils im Einzelnen aufzuführen. Wenn inhaltliche Aspekte beleuchtet werden, die von ihm in seiner Studie nicht berücksichtigt wurden oder Präzisierungen vorgenommen werden, sind die jeweiligen Quellen aufgeführt.

239 So Mosberg, REeducation, S. 143. 
munistischen Vorzeichen die Werte der liberalen Demokratie amerikanischer Provenienz in die Zonen Nachkriegsdeutschlands vermittelt werden sollten. ${ }^{240}$ Die Organisation lag in den Händen der eigens gegründeten Political Information Branch (PIB) der Information Control Division (ICD), die zuerst der Militärregierung (OMGUS) und später der Hohen Kommission (HICOG) unter der Führung von McCloy unterstand.

Vor dem Hintergrund des Kalten Krieges veränderte sich die amerikanische Deutschlandpolitik insofern, dass fortan eine Politik der »Re-Orientation« einsetzte, die die demokratischen Grundtendenzen der Reeducation aufgriff und sie der neuen (internationalen) politischen Situation anpasste. Nach dem Dafürhalten der Amerikaner bestand angesichts des sowjetischen Bedrohungspotenzials die Notwendigkeit, Westdeutschland $\mathrm{zu}$ einem strategisch wichtigen Bündnispartner $\mathrm{zu}$ machen und infolgedessen auch dessen Bevölkerung dauerhaft und tief greifend für die »eigene Sache « zu gewinnen, sodass im Hinblick auf die Umerziehung der Deutschen ein flexiblerer Ansatz vonnöten war. Daraus folgte, dass das grundsätzliche Ziel einer tief greifenden gesellschaftlichen Demokratisierung erhalten blieb, wobei man allerdings der fundamentalen politischen Auseinandersetzung mit der Sowjetunion Rechnung trug. Demgegenüber wurde der genuinen Analyse des nationalsozialistischen Herrschaftssystems und der -praxis sowie deren Bedingungen und Ursachen kein überragender Stellenwert eingeräumt. ${ }^{241}$

Selbstverständlich ging es den Amerikanern darum, dass ihre Umerziehungspolitik von einer breiten Akzeptanz in der deutschen Bevölkerung getragen wird, sodass ab Oktober 1947 auch die amerikanische Kulturpolitik einen deutlich antikommunistischen Kurs einschlug. ${ }^{242} \mathrm{Zu}$ diesem Zeitpunkt kamen die ersten konzeptionellen Überlegungen für den Monat in Gang, der als ein anspruchsvolles, propagandistisch ausgerichtetes Instrument amerikanischer Wertevermittlung in Deutschland gedacht war und mit dem auf hohem Niveau eine antikommunistische Diskussion geführt werden sollte, die auch die generelle Auseinandersetzung mit »nationaldeutschen Werten« beinhaltete. ${ }^{243}$

Das heißt, letzten Endes war Der Monat ein Organ liberaldemokratischer und antikommunistischer Propaganda, die keineswegs undifferenziert und polemisch war, sondern eine erhebliche intellektuelle Kapazität aufwies. Gleichermaßen war er auch ein Instrument der »Reorientation« mit ihrem doppelten Bezugssystem, so Michael Hochgeschwender:

Einerseits sollten die Deutschen, jetzt verstanden als prinzipiell von der NSDAP zu unterscheidende - anthropologisch optimistische eingeschätzte - Größe, als antikommunistische Bündnispartner gewonnen werden, andererseits diente »Der Monat« der subtilen Vergangenheitsbewältigung, indem er mit Hilfe soziologischer, psychologischer, historischer und philosophischer Analysen deutsche geistesgeschichtliche Traditionsbestände zu destruieren oder liberal umzuinterpretieren suchte. ${ }^{244}$

240 Vgl. Harold Hurwitz, Die Stunde Null der deutschen Presse. Die amerikanische Pressepolitik in Deutschland 1945-1949, Köln 1972, S. 333-339.

241 Hochgeschwender, Freiheit in der Offensive?, S. 145.

242 Ebd., S. 126

243 Ebd., S. 129.

244 Ebd., S. $149 \mathrm{f}$. 
Ebendiese beiden Aspekte der »Reorientation« sollten durch die Verbreitung ausländischer, insbesondere angelsächsischer Literatur erreicht werden, die während der Phase der »Hitler-Diktatur « für die Deutschen nicht zugänglich waren. Hinzu kamen zwei weitere Aspekte, die von grundlegender Bedeutung waren. Angesichts der bei der Operation »Talk Back« vorherrschenden Auffassung, dass der Kalte Krieg auf der Seite der politischen Linken (SPD, Gewerkschaften und Linksintellektuellen) entschieden würde, zielte der Monat auch auf eine westorientierte Reform der SPD und auf die antitotalitäre politische Einvernahme der westdeutschen Intellektuellen. Diese vier Bestandteile sind für Hochgeschwender jeweils zusammenzusehen, da für ihn die Zeitschrift ein Instrument anspruchsvoller intellektueller Propaganda des Westens im Kalten Krieg war - und als ein solches auch von Lasky in seiner Doppelfunktion als Herausgeber und Chefredakteur geplant war. "Gerade weil >Der Monat«", so der Tübinger Historiker in diesem Zusammenhang,

mit seinem Bemühen, die Deutschen auch ideell im Westen zu verankern, etwas weitgehend Neues in der deutschen Ceistesgeschichte anstrebte, vermochten die Redaktionsangehörigen Forderungen nach einer antifaschistischen Front zur radikalen Umgestaltung der deutschen Cesellschaft nicht anders zu interpretieren denn als stalinistische Propagandaattacken. Selbst ernsthafte Konzepte einer sozialistischen Neugestaltung Deutschlands mußten fast zwangsläufig unter dieses Verdikt fallen. ${ }^{245}$

Der Monat war also ein unmittelbares Ergebnis der von Clay initiierten antikommunistischen Operation »Talk Back«, und aller Voraussicht nach ist die Idee zu der Zeitschrift direkt in der PIB entwickelt worden. Möglicherweise ist sogar Clay persönlich auf den Gedanken gekommen und hat anschließend Lasky für das Projekt gewonnen - nachdem er auf ihn durch dessen Auftritt auf dem Ersten Deutschen Schriftstellerkongress aufmerksam wurde. ${ }^{246}$ Allerdings behauptet Lasky wiederum, dass die Idee für den Monat von ihm selbst stammte. ${ }^{247}$ Letzten Endes war die Bedeutung der geistigen Urheberschaft der Zeitschrift für die tatsächliche Planung und Ausgestaltung des Monat nicht entscheidend, da diese von Anbeginn in den Händen von Lasky lag. Unterstand die Zeitschrift anfangs dem PIB im Rahmen der ICD (später ISD), so nach dem Übergang zur HICOG dem Office of Public Affairs. Allerdings war das Zeitschriftenprojekt innerhalb der US-amerikanischen Besatzungsbehörden zu Beginn keinesfalls unumstritten. Bedenken gegen die »Lasky-Zeitschrift« wurden in erster Linie vom New York Field Office (NYFO) vom War Department artikuliert. In diesem Zusammenhang waren es vor allem Ruben S. Nathan und Paul Kecskemeti, die ihre politisch motivierte Skepsis vorbrachten, da sie den Verdacht hatten, dass Clay und Lasky gegenüber den Deutschen einen entspannteren Kurs fahren würden, als es das bis dahin gültige Reeducation-Konzept vorsah. Ihrer Auffassung nach bestand die Gefahr, dass anstatt einer offensiven Umerziehung bei gleichzeitigem defensivem Antikommunismus ein offensiver Antikommunismus an die Stelle tritt, der möglicherweise die Reeducationund Reorientation-Politik gefährden konnte. Tatsächlich hatten sich angesichts des Kalten Krieges innerhalb der US-Administration mehr und mehr die Personen bzw.

245 Ebd., S. 150.

246 Ebd.

247 So Lasky in Martin, Orwell, Koestler und all die anderen, S. 23. 
Kräfte durchgesetzt, die einen neuen Blick auf die Deutschen warfen und den ehemaligen Kriegsgegner zum »Partner « machen wollten. In diesem politischen Fahrwasser befand sich auch Lasky, der zwar keineswegs die Auseinandersetzung mit der Geschichte des >Dritten Reiches` abschwächen wollte, allerdings die zeitgenössischen Gefahren durch den Stalinismus für größer hielt als von einem, so seine Auffassung, »längst überwundenen Faschismus«. In diesem Konflikt um den Monat ging es mithin auch um verschiedene Vorstellungen im Hinblick auf die zukünftige Deutschlandpolitik innerhalb der amerikanischen Militärregierung: Plädierten die Vertreter der »OMGUS-Linken« bzw. die linken »New Dealer«, zu denen zahlreiche Emigranten wie zum Beispiel Paul Kecskemeti zählten, für härtere Maßnahmen gegenüber den Deutschen, sprachen sich die Anhänger Trumans, wie General Clay und Lasky, für eine Politik aus, die unter dem Primat des Antikommunismus stehen sollte. Erst mit dem Sieg Trumans bei den Präsidentschaftswahlen 1948 war dieser Konflikt endgültig zugunsten der Antikommunisten entschieden, obwohl die »OMGUS-Linke« bereits seit 1946 in Bezug auf die amerikanische Besatzungspolitik mehr und mehr Einfluss verloren hatte. ${ }^{248}$ Vor diesem Hintergrund konnten sich Clay und Lasky auch mit dem Projekt Der Monat gegen die Vorbehalte seitens der NYFO durchsetzen. Im Oktober 1948 erschien dann die erste Ausgabe des Monat und avancierte unter der Ägide von Lasky ${ }^{249}$ bei einer durchschnittlichen Auflagenzahl von knapp 25000 Exemplaren in der Mitte der 1950er-Jahre nicht nur zu einer der einflussreichsten Zeitschrift der deutschen Nachkriegsgeschichte. ${ }^{250}$ Für den renommierten Historiker und Journalisten Walter Laqueur war Der Monat in den 1950er- und 1960er-Jahren sogar »die bedeutendste Zeitschrift Europas, ja wahrscheinlich der ganzen Welt ${ }^{251}$.

Lasky wurde am 15. Januar 1920 als Sohn jüdisch-russischer Emigranten in New York geboren und starb im Alter von 84 Jahren im Mai 2004 in Berlin. Sein Vater war ein kleiner Textilunternehmer. Er studierte in den 1930er-Jahren unter anderem am berühmten New York City College, das damals eine Hochburg trotzkistischer Intellek-

248 Siehe Michael Hochgeschwender, Remigranten im Umfeld der Zeitschrift Der Monat und des Congress for Cultural Freedom (CCF), in: Krohn/Schildt, Zwischen den Stühlen?, S. 180-206, hier S. 186 f.

249 Lasky fungierte von Oktober 1948 (H. 1) bis April 1960 (H. 139) als Herausgeber und Chefredakteur des Monat (ab 1958 wurde er Nachfolger von Irving Kristol als Herausgeber des Londoner Encounter). Lasky wurde durch den Schweizer Publizisten Fritz René Allemann ersetzt, der fortan mit Hellmut Jaesrich gemeinsam den Monat herausbrachte und ab Februar 1961 auch Miteigentümer der Zeitschrift wurde. Im Mai 1962 (H. 164) kam der deutsche Schriftsteller Peter Härtling als Mitherausgeber dazu. Nachdem Allemann sich im August 1964 (H. 191) als Mitherausgeber der Zeitschrift verabschiedete, dauerte es bis zum Januar 1967 (H. 220), bis der deutsche Journalist und Schriftsteller Klaus Harpprecht an seine Stelle trat. Harpprecht übernahm mit Beginn des Jahres 1969 (H. 244) -in Zusammenarbeit mit dem finanziellen Unterstützer, dem Bucerius Zeit-Verlag-die »alleinige Verantwortung « (Marko Martin) für die Zeitschrift und wurde von den beiden Redakteuren Peter Härtling und Hellmut Jaesrich unterstützt. Fortan wurde die Zeitschrift unter dem Titel Monat herausgegeben und erschien bis März 1971 (H. 270). Danach wurde Der Monat im Oktober 1978 wiederbelebt und erschien vierteljährlich bis 1986, wobei Melvin J. Lasky, Michael Naumann und Helga Hegewisch als Herausgeber fungierten.

250 Diese Auflagenzahl pendelte sich Mitte der 1950er-Jahre ein. Hochgeschwender (Freiheit in der Offensive?, S. 199, Anm. 161) weist daraufhin, dass Der Monat damit die "größte kulturpolitische Zeitschrift Europas« war; dieser Erfolg wurde erst durch den Encounter erheblich übertroffen, der indes auch auf den nordamerikanischen Markt zielte.

251 Zit. n. Martin, Orwell, Koestler und all die anderen, S. 99. 
tueller war. Nachdem auch Lasky zwischenzeitlich zu einem Anhänger des Trotzkismus avancierte, entwickelte er sich allerdings alsbald zu einem linken Antikommunisten. Seine berufliche Laufbahn begann er als Redakteur der New Yorker Zeitschrift New Leader, ein Diskussionsforum der unabhängigen intellektuellen Linken, für die er von 1942 bis 1944 arbeitete, und diente danach als Captain und Kriegshistoriker in der amerikanischen Armee. Nach Kriegsende kam er als Kulturoffizier nach Berlin und schrieb als Korrespondent für New Leader und Partisan Review. Angesichts des Kampfes mit dem sowjetischen Herrschaftsregime unter Stalin trat Lasky für eine amerikanische Außenpolitik ein, die Deutschland und die Bevölkerung zu einem Verbündeten machen sollte, und vertrat in Bezug auf die Auseinandersetzung mit dem Nationalsozialismus die Auffassung, dass zwischen dem eigentlichen »Regime«, d. h. der unmittelbaren Führung, und dem »Volk« klar getrennt werden müsse.

Als Mitherausgeber des Monat fungierte der am 17. September 1908 in Berlin geborene (und 1989 verstorbene) Hellmut Jaesrich. Er hatte in Berlin, Grenoble, Paris und Heidelberg Neuphilologie, Geschichte und Philosophie studiert und promovierte im Anschluss daran bei Ernst Robert Curtius. Nach dem Ende des >Dritten Reiches arbeitete er als Redakteur in der von Alfred Kantorowicz herausgegebenen Kulturzeitschrift Sie. Jaesrich, der später als »literarische[s] Genie« (Hermann Glaser) bezeichnet wurde, brachte in die international und kosmopolitisch ausgerichtete Zeitschrift, insbesondere was die Autorenauswahl anbelangte, eine spezifisch deutsche Note ein. ${ }^{252}$

Obwohl Der Monat in den ersten Jahren in München gedruckt wurde, befanden sich die Redaktionsräume aus guten Gründen in Berlin. - Berlin war nicht zuletzt nach der sowjetischen Blockade gewissermaßen das Zentrum des Kalten Krieges und die bedeutendste Schnittstelle zwischen den beiden Blöcken. Durch die Blockade bekam sie den Charakter der »Frontstadt « im Kalten Krieg und des Bollwerkes gegen den Kommunismus, und die Blockadeerlebnisse gaben der antikommunistischen Propaganda konkrete Nahrung. Berlin mit seinen Einwohnern war der lebendige Beweis für den Freiheitswillen der Deutschen und mithin für die Richtigkeit des »Reorientation«-Ansatzes. Zudem hatte sich in der ehemaligen und nahezu fast vollkommen zerstörten Reichshauptstadt seit Sommer 1945 ein blühendes Kulturleben entwickelt, ohne indes an das überragende Niveau der Weimarer Republik heranzureichen. Vor diesem Hintergrund drängte sich Berlin als Standort für eine intellektuelle Zeitschrift in Deutschland mit internationalem Profil und Renommee nahezu auf. Und in diesem Zusammenhang spielten auch die anvisierten Lesergruppen eine wesentliche Rolle: Der Monat wollte in erster Linie Intellektuelle, das Bildungsbürgertum, Schüler, Studenten, konfessionell nichtgebundene oder liberale Protestanten sowie Sozialdemokraten ansprechen, d.h. Rezipienten mit potenziellen Residualzonen sowohl kleindeutsch-nationalneutralistischer als auch westlich-aufgeklärter Traditionen. Vor allem für diese Gruppen war Berlin mehr als jede andere Stadt der amerikanischen Besatzungszone ein Ort, dessen kulturelles Leben man sehr genau verfolgte. ${ }^{253}$

252 Siehe Hochgeschwender, Freiheit in der Offensive?, S. 150 f. Neben den beiden Herausgebern Lasky und Jaesrich verfügte die Zeitschrift im Jahre 1952 über eine Redaktion, der vier festangestellte Redakteure angehörten: Hans R. Hentschel, Alfred Kellner, Erik Nohara und Wolfgang Stiebler (ebd., S. 160).

253 Siehe ebd., S. $153 \mathrm{f}$. 
Dass Lasky die Funktion des Herausgebers des Monat übernahm, mag mehrere Gründe gehabt haben. Allerdings besaß er einen ganz entscheidenden Vorteil. Er war prädestiniert, ein zentrales Ziel im Rahmen der amerikanischen Grundausrichtung zu realisieren: nämlich vor allem deutsche Intellektuelle für die »westliche Seite« zu gewinnen und in die antikommunistischen Aktivitäten einzubeziehen. Denn Lasky kannte nahezu jeden und seit seinem spektakulären Auftritt auf dem Ersten Deutschen Schriftstellerkongress kannten ihn die allermeisten deutschen Intellektuellen. Hierbei konnte er auf bereits bestehende Netzwerke zurückgreifen. Beispielsweise war Lasky seit dem Kongress im Oktober 1947 über Günther Birkenfeld mit einigen Vertretern der antikommunistischen Inneren Emigration verbunden, sodass er beispielsweise Rudolf Hagelstange, Stefan Andres, Rudolf Pechel, Friedrich Luft als Autoren bzw. Mitarbeiter einbeziehen konnte. Von nicht geringer Bedeutung war auch, dass Lasky mit der Heidelberger Gruppe um die Zeitschrift Die Wandlung verbunden war, sodass er die Kontakte von Alfred Weber oder auch Dolf Sternberger nutzen konnte. Zudem war er mit Golo Mann, Karl Jaspers und Hannah Arendt bestens bekannt. Über Berlins Bürgermeister Ernst Reuter knüpfte Lasky im Laufe der Zeit Kontakte unter anderem zu SPD-Kreisen der Stadt - wie zum Beispiel zu Willy Brandt. ${ }^{254}$

Durch seine politische Herkunft und seine redaktionellen Aktivitäten in den beiden Zeitschriften New Leader und Partisan Review konnte Lasky mehrere Exponenten aus dem Umfeld der prominenten »New York Intellectuals« zur Mitarbeit im Monat gewinnen, die ebenfalls eine trotzkistische Vergangenheit besaßen und nach ihrer Auseinandersetzung mit dem Stalinismus seinen konsequenten Antikommunismus teilten. $\mathrm{Zu}$ nennen sind hier insbesondere Sidney Hook, James Burnham, Lionel Trilling und Dwight Macdonald, ${ }^{255}$ die in dem Periodikum veröffentlichten. Und von überragender Bedeutung sollte vor allem die Bekanntschaft mit Arthur Koestler sein, der - durch seine wichtige Rolle im Kontext der Vorgeschichte des »Kongresses für kulturelle Freiheit« - auf ein transnationales Netzwerk linksliberaler, antikommunistischer Intellektueller zurückgreifen konnte. Erstens hatte Koestler zusammen mit George Orwell im Rahmen der seit 1941 in England erscheinenden antikommunistischen Zeitschrift Horizon nicht nur Kontakte zu führenden intellektuellen Persönlichkeiten des Landes wie zum Beispiel Victor Gollancz oder Michael Polanyi -, sondern auch zu führenden antikommunistischen Mitgliedern der Labour Partei wie Denis Healey oder Richard H. S. Crossmann. Zweitens kannte Koestler seit Langem den früheren Anhänger des Kommunismus Manès Sperber, ${ }^{256}$ der wiederum die Gesprächsfäden zu anderen spä-

254 Siehe ebd., S. 153-156.

255 Der ehemalige Trotzkist Dwight Macdonald war mit Lasky befreundet und nicht nur Mitherausgeber der Zeitschrift Partisan Review, sondern brachte auch die in den Vereinigten Staaten einflussreiche Zeitschrift Politics heraus, die von 1944 bis 1949 erschien. Diese Zeitschrift gehört, was die personelle, inhaltlich und politische Ausrichtung anbelangt, durchaus zur Vorgeschichte des Monat. Siehe zu Politics die entsprechenden Ausführungen in Kap. II.3.1.

256 Manès Sperber (1905-1984) gehörte, nachdem er 1927 in die KPD eintrat und 1933 von den Nationalsozialisten vorübergehend verhaftet wurde, zum engsten Mitarbeiterstab des »Münzenberg-Apparates«. Hier arbeitete er von 1934 bis 1936 im Auftrag der Partei für das von der Komintern gegründete Institut zum Studium des Faschismus und für den Weltjugendkongreß gegen den Krieg und Faschismus. Nachdem Sperber angesichts der Moskauer Schauprozesse 1938 aus der KP austrat - in diesem Jahr veröffentlichte er in Paris seine sozialpsychologische Studie Zur Analyse der Tyrannis - gehörte u. a. mit Koestler und Löwenthal zum redaktionellen Kommitee der von Münzen- 
teren Autoren des Monat knüpfen konnte; dem Renegaten gelang es beispielsweise, die einflussreichen französischen Intellektuellen Raymond Aron, André Malraux und Albert Camus einzubeziehen. ${ }^{257}$

Nicht zuletzt durch die genannten persönlichen Kontakte von Lasky und bereits bestehender Netzwerke von Intellektuellen konnte Der Monat also nach dem Erscheinen der ersten Ausgabe auf zahlreiche Autoren aus dem deutschsprachigen Raum und aus dem Ausland zurückgreifen. So veröffentlichten im Laufe der Jahre - neben den bereits aufgeführten Personen - deutsche Exilliteraten (wie Thomas Mann, Hans Sahl, Hermann Kesten, Peter de Mendelssohn oder Hilde Spiel), ehemalige Anhänger des Kommunismus (so Margarete Buber-Neumann, Ruth Fischer, Ernest Salter, Franz Borkenau, Richard Löwenthal, Milovan Djilas, André Gide oder Ignazio Silone), literarische Größen der Zeit (zum Beispiel T. S. Eliot, William Faulkner, Ernest Hemingway, Christopher Fry, Thornton Wilder, Aldous Huxley, George Bernhard Shaw), Philosophen, Ökonomen oder Wissenschaftler und Historiker (wie Theodor W. Adorno, Max Horkheimer, Wilhelm Röpke, Joseph A. Schumpeter, Bertrand Russell, Hugh R. Trevor-Roper, Alan Bullock oder Walther Hofer).

Den Schwerpunkt der Autoren des Monat - zumindest für die 1950er-Jahre - bildeten aber Mitarbeiter des »Kongresses für kulturelle Freiheiten«, deren Namen zum Teil bereits genannt wurden: Neben Hannah Arendt und Raymond Aron waren dies in erster Linie Arthur Koestler, Ignazio Silone, Franz Borkenau, John Dewey, Benedetto Croce, Czeslaw Milosz, Salvador de Madariaga, Arthur M. Schlesinger jr., Michael Polanyi, Peter de Mendelssohn, Denis de Rougemont und nicht zuletzt der Herausgeber der französischen Schwesterzeitschrift Preuves, François Bondy. Aus der deutschen Sektion des »Kongresses « und aus dessen internationalen Umfeld stießen hinzu: Rudolf Hagelstange, Carl Linfert, Theodor Plivier sowie unter anderem Karl Jaspers, Richard Löwenthal, Hans Schwab-Felisch, Ernest Salter, Ludwig Marcuse, Alfred Weber, Norbert Mühlen, Ernst Reuter und Herbert Lüthy. ${ }^{258}$

Sowohl Der Monat als auch der »Kongreß für kulturelle Freiheit« waren spezielle Instrumente der amerikanischen Außenpolitik nach dem Ende des Zweiten Weltkrieges. Sie verdankten ihre Entstehung den Entstehungsbedingungen des Kalten Krieges und waren organisatorisch, personell, inhaltlich und funktional nicht eindeutig voneinander zu trennen. Beide Institutionen stellten - allgemein ausgedrückt - den Versuch dar, im Systemkonflikt mit der Sowjetunion das liberaldemokratisch-kapitalistische System der Vereinigten Staaten politisch und kulturell zu stabilisieren. Während allerdings die "Lasky-Zeitschrift« in erster Linie auf Deutschland ausgerichtet war und nicht zuletzt vor dem Hintergrund der amerikanischen Besatzungspolitik $\mathrm{zu}$ verstehen ist, war der »Kongreß« von vornherein weltweit konzipiert. Gleichwohl entfaltete der »Kongreß«, dessen Zentrale sich in Paris befand, seine Aktivitäten ins-

berg herausgegebenen Zeitschrift Die Zukunft, die inhaltlich, politisch und personell ebenso zur Vorgeschichte des Monat gehört. Siehe zu Die Zukunft die entsprechenden Ausführungen in Kap. II.3.1. Nach dem Zweiten Weltkrieg besaß Sperber eine wichtige Funktion beim 1950 gegründeten »Kongreß für kulturelle Freiheit«(siehe Kap. I.5) und gehörte dem internationalen Exekutivkomitee dieser Institution an.

257 Vgl. im Einzelnen allerdings mit Blick auf die Vorgeschichte des »Kongresses für kulturelle Freiheit«, ebd., S. 113-118.

258 Siehe ebd., S. 174. Gemeinhin besaßen neben ehemaligen Nationalsozialisten auch Faschisten, Kommunisten und Nationalisten keine Möglichkeit, im Monat zu veröfflichten, vgl. ebd., S. 171. 
besondere in den unmittelbaren Nachkriegsjahren angesichts des Eindrucks der unmittelbaren Bedrohung durch den Stalinismus und der tatsächlichen Stärke der kommunistischen Parteien in Frankreich und Italien, in Europa. Insofern gab es auch von Anbeginn eine finanzielle Abhängigkeit der Zeitschrift und des »Kongresses« von USamerikanischen Stellen. Während der »Kongreß «ornehmlich von finanziellen Mitteln der CIA abhing, die anfangs direkt und später unter strengster Geheimhaltung über die sogenannten dummy foundations ausgezahlt wurden, war die Finanzierung des Monat ein wenig komplexer und ist in Bezug auf die Frage des Geldgebers in der neuesten Forschung durchaus umstritten.

Die britische Literaturwissenschaftlerin Frances Stonor Saunders vertritt in ihrer Studie Wer die Zeche zahlt ...: Der CIA und die Kultur im Kalten Krieg die sehr allgemein gehaltene Auffassung, die ohne Quellenangaben erfolgt, dass die Zeitschrift im Laufe der Jahre zuerst aus >vertraulichen Fonds` des Marshallplans, dann der CIA, danach durch Mittel von der Ford Foundation und anschließend wieder durch CIA-Gelder finanziert wurde. ${ }^{259}$ Michael Hochgeschwender sieht in seiner Studie Freiheit in der Offensive? Der Kongreß für kulturelle Freiheit und die Deutschen die Sache der Finanzierung des Monat differenzierter. Für ihn stand die Finanzierung jeweils im Kontext des sich im Laufe der Jahre verändernden rechtlichen Status. ${ }^{260}$ Hochgeschwenders Meinung nach - die er durch zahlreiche Quellen belegen kann - erhielt Der Monat durch die Tatsache, dass er von der US-amerikanischen Militärregierung gegründet wurde, anfangs seine finanziellen Mittel direkt von OMGUS und später von der HICOG. ${ }^{261} \mathrm{Ab}$ August 1954 leiteten Lasky und Jaesrich durch die Gründung der sogenannten Gesellschaft für internationale Publizistik m. b. H. mit Sitz in Berlin die Privatisierung des Monat ein, wobei das Stammkapital in Höhe von 40000 DM jeweils zur Hälfte bei den beiden Herausgebern lag. Nachdem sie im September von amerikanischer Stelle eine neue Lizenz erhalten hatten, verfügte der Monat ab Oktober 1954 mit der Ford Foundation über einen finanzstarken Partner und ging endgültig in private Hände über. Bis Ende Juni 1958 flossen finanzielle Mittel der Ford Foundation, die dann ohne erkennbare Gründe ihre Zahlungen einstellte. Ab Juli 1958 wiederum trat die Zentrale des »Kongresses für kulturelle Freiheit« in Paris als Geldgeber in Erscheinung, die bis 1963 als Spender auftrat. Für Hochgeschwender folgte daraus, dass der Monat erst ab Juli 1958 von der CIA Geld erhalten hatte, im Gegensatz zum Beispiel zu den Schwesterzeitschriften Encounter und Preuves, die von Anbeginn durch den amerikanischen Geheimdienst in Form von verdeckt gezahlten Geldern finanziert wurden. ${ }^{262}$

Die Frage, inwiefern Der Monat von Anbeginn auch finanzielle Zuwendungen der CIA erhalten hat, kann hier nicht weiter geklärt werden und muss letzten Endes offen bleiben. Gleichwohl gibt es einige Anhaltspunkte dafür, dass die »Lasky-Zeitschrift« ebenfalls bereits vor Juli 1958 auch durch CIA-Gelder (mit-)finanziert wurde: nämlich seit Oktober 1948 aus »Vertraulichen Fonds« des Marshallplanes (bzw. der ame-

259 Saunders, Wer die Zeche zahlt ..., S. 41.

260 Das Folgende nach Hochgeschwender, Freiheit in der Offensive?, S. 159-170.

261 Aus diesen Gründen konnte der Verkaufspreis der Zeitschrift bis Oktober 1954 niedrig gehalten werden: Sie kostete pro Exemplar 1,00 DM, danach 1,50 DM. Ab April 1961 erhöhte sich der Verkaufspreis auf 2,00 DM, bis dann im April 1964 eine Erhöhung um 50 Pfennig einsetzte. Die letzte Preiserhöhung erfolgte im Januar 1969 - fortan kostete das einzelne Heft 3,00 DM.

262 Hochgeschwender, Freiheit in der Offensive?, S. 166. 
rikanischen Besatzungsbehörden) und ab Oktober 1954 durch die Ford Foundation; in beiden Fällen lief dann die Finanzierung in Form von verdeckt gezahlten Geldern des amerikanischen Geheimdienstes. Möglicherweise flossen auch bereits vor Juli 1958 über den »Kongreß für kulturelle Freiheit« geheim gehaltene Gelder, der bekanntlich mit dem Monat assoziiert war und unter anderem die gesamten 1950er-Jahre zu dem Organ überaus intensive Beziehungen pflegte, sodass beispielsweise Lasky und Jaesrich regelmäßig an den Herausgebertreffen der wichtigsten »Kongreß«-Zeitschriften teilnahmen. Da aber der »Kongreß « vornehmlich von finanziellen Mitteln der CIA abhing, die anfangs direkt und später unter strengster Geheimhaltung über die »dummy foundations « - wie zum Beispiel der Ford Foundation - ausgezahlt wurden, wäre eine frühere Beteiligung der CIA beim Monat durchaus denkbar. Solange aber der genaue Zeitpunkt für die direkte oder indirekte finanzielle Beteiligung der CIA am Monat vor allem nicht durch Dokumente geklärt werden kann, kann dieser zweifelsohne wichtige Aspekt nicht endgültig entschieden werden. Fest steht nur, dass die CIA den Monat finanziell unterstützte. Dies wurde auch vom späteren Mitherausgeber Klaus Harpprecht bestätigt, der meinte, dass die geheime Finanzierung über die CIA aus haushaltstechnischen Gründen geschah, "weil die Abgeordneten und Senatoren des amerikanischen Kongresses kaum bereit gewesen wären, die Mittel für solchen Luxus $\mathrm{zu}$ genehmigen $\aleph^{263}$. Allerdings machten die Herausgeber des Monat überhaupt kein Geheimnis daraus, dass das Organ im Namen der US-amerikanischen Besatzungsbehörden herausgegeben wurde: Dies war monatlich im Impressum zu lesen und wurde von Lasky und Jaesrich im Rahmen der sogenannten Privatisierung der Zeitschrift im Septemberheft 1954 den Lesern auch ausdrücklich mitgeteilt. ${ }^{264}$

\subsection{Die inhaltliche Ausrichtung des Monat unter der Ägide Laskys}

Die inhaltliche Ausrichtung des Monat von Oktober 1948 bis April 1960 (H. I bis 139) stand ohne Zweifel in einem unmittelbaren Zusammenhang mit der speziellen Funktion der Zeitschrift in diesem Zeitraum. Hierbei lag in den ersten Jahren im Großen und Ganzen keine direkte Einflussnahme seitens der offiziellen amerikanischen Stellen vor. Nach Auffassung von Harold Hurwitz besaß Der Monat als »offizielles Organ« ungewöhnlich viel Freiheit, weil er gewisse Grenzen respektierte. ${ }^{265}$ Nach der Ablösung von staatlichen Stellen und dem Übergang 1954 zur Ford Foundation und 1958 zum »Kongreß für kulturelle Freiheit« hatte das Organ um Lasky ungewöhnlich viel Freiheit, weil er sowohl die inhaltliche Gesamtkonzeption als auch die Grenzen selbst festlegte.

Im Kern gruppierte sich die inhaltliche Ausrichtung der Zeitschrift um die beiden ideologisch aufgeladenen Themenfelder, die für die Gründung des Monat von heraus-

263 Zit. n. Martin, Orwell, Koestler und all die anderen, S. 21.

264 Siehe Der Monat 6 (1954), H. 72, Umschlagseite innen. Zudem räumte Jaesrich im Mai 1955 (Der Monat 7 [1955], H. 80, S. 180) ein, dass die Zeitschrift direkt von der Ford Foundation, einer sogenannten privaten Stiftung, subventioniert wurde. Vgl. in diesem Zusammenhang auch $\mathrm{H}$ [ellmut] ][aesrich], Das Besatzungskind, in: Der Monat 20 (1968), H. 241, S. 4; Gerd Bucerius, Liebe Leser!, in: Der Monat 21 (1969), H. 244, S. 6 f., sowie ders., Noch auf ein Wort, in: Der Monat 23 (1971), H. 270, S. 4 f.

265 Harold Hurwitz, Der heimliche Leser. Beiträge zur Soziologie des geistigen Widerstandes, Köln/Berlin 1966, S. 19. 